\title{
ON THE ACCURACY OF DETERMINING THE DISPERSION MEASURE FOR PSR 0809+74 USING MULTIFREQUENCY OBSERVATIONS
}

\author{
YU. M. BRUCK AND O. M. Ulyanov \\ Institute of Radio Astronomy
}

A question on the accuracy of determining the dispersion measure $D M$ for PSR $0809+74$ arises in connection with the discussion which has developed in recent years concerning observations of the effect of the superdispersion time delay (STD) (Shitov 1985, Shitov, Malofeev, and Izvekova 1988, Kuz'min 1985a) attributed to the twisting of the magnetic field lines or to, competing with it as to the observation manifestations, the aberration effect (Cordes 1978, Matese and Whitmire 1980, Ulyanov 1989, Ulyanov 1990). An interpretation of the available data in favor of any of these effects would be inappropriate without a quantitative analysis of the observation errors. With our aim to define the $D M$ value and its errors more precisely, we shall analyze both the observational data available elsewhere and our results for PSR 0809+74 which, from the observational data in Shitov (1985), Shitov et al. (1986), and Kuz'min et al. (1985), has the most pronounced STD effect.

Until now the most precise determinations of $D M$ for PSR $0809+74$ have been achieved using twofrequency $\left(\nu_{1}=102.57 \mathrm{MHz}, \nu_{2}=101 \mathrm{MHz}\right.$ ) observations of microstructure (Smirnova et al. 1986) where $D M=5.751 \pm 0.003 \mathrm{pc} \mathrm{cm}^{-3}$. This estimation is made using time-delay measurements of the propagation of microstructure between frequencies $\nu_{1}$ and $\nu_{2}$. Herewith we follow Kardashev et al. (1982) and Boriakoff (1983) in suggesting that the correlating part of the micropulse radiation follows the dispersion law. As shown by Ulyanov (1990), the effects of aberration and twisting cannot be separated when measuring time delays at two frequencies.

Let us estimate the DM of PSR $0809+74$ using the results of Smirnova et al. (1986) and our own observations at the frequencies $\nu_{3}=25.151 \mathrm{MHz}$ and $\nu_{4}=16.699 \mathrm{MHz}$ which were conducted during the night hours on 5 and 6 November 1982 (figure 1). To estimate the $D M$ from the observational results over the two frequency ranges (and taking into account the aberration effect), we use the following formula from Ulyanov (1990):

$$
D M_{\text {real }}=\frac{2.41}{1 / \nu_{2}^{2}-1 / \nu_{1}^{2}}\left\{\tau\left(\nu_{1}, \nu_{2}\right)+\frac{\left[1-\left(\nu_{1} / \nu_{2}\right)^{-2 / 3}\right]\left[\tau\left(\nu_{1}, \nu_{2}\right)-b \tau\left(\nu_{3}, \nu_{4}\right)\right]}{\left\{b\left(\nu_{2} / \nu_{4}\right)^{2 / 3}\left[1-\left(\nu_{3} / \nu_{4}\right)^{-2 / 3}\right]-\left[1-\left(\nu_{1} / \nu_{2}\right)^{-2 / 3}\right]\right\}}\right\}
$$

where the $D M$ is the real dispersion measure accounting for the aberration effect, $\nu_{1}>\nu_{2}>\nu_{3}>\nu_{4}$ are the observation frequencies in units of $100 \mathrm{MHz}, b=\left(1 / \nu_{2}^{2}-1 / \nu_{1}^{2}\right) /\left(1 / \nu_{4}^{2}-1 / \nu_{3}^{2}\right), \tau\left(\nu_{i}, \nu_{j}\right)$ is the visible time delay of the pulsar radiofrequency radiation (PRR) between the frequencies $\nu_{i}$ and $\nu_{j}$. Here we neglect the twist effect of the pulsar magnetic field since it is an order of magnitude less than the aberration effect (Ulyanov 1990).

The observational results which we analyze were conducted in different years. Nevertheless their use in the $D M$ analysis of eq.(1) is valid as we want to determine the average $D M$ value for the interstellar medium and to estimate its error; for this, it is enough to know the absolute time delays between the different frequencies and the errors in both types of observations. The corrections to the $D M$ due to the different relative velocities of the observer and the source-i.e. the corrections for the Doppler effectwill be considerably smaller than the instrumental corrections for this pulsar and later on they will not be taken into account. As follows from Smirnova et al. (1986), the absolute time delay between the frequencies $\nu_{1}=102.56 \mathrm{MHz}$ and $\nu_{2}=101 \mathrm{MHz}$ is $0.071065 \mathrm{~s}$. $\left[\tau\left(\nu_{1}, \nu_{2}\right)=0.071065 \mathrm{~s}\right]$. For our observations (see figure 1), the absolute time delay is $\tau_{\mathrm{A}}\left(\nu_{3}, \nu_{4}\right)=47.875896 \mathrm{~s}$ if the points (A) of the radiation minima in the main pulse window are taken as fiducial ones or $\tau_{B}\left(\nu_{3}, \nu_{4}\right)=47.9374 \mathrm{~s}$ if the points (B) of the radiation maxima are taken as fiducial. Then the calculation using eq.(1) yields:

$$
D M(\mathrm{~A})_{\mathrm{real}}=5.754 \mathrm{pccm}^{-3} \text { or } D M(\mathrm{~B})_{\mathrm{resl}}=5.760 \mathrm{pc} \mathrm{cm}^{-3}
$$

The error in these values is the same as it results from two factors. First, it is caused by the instrumental error, (i.e. by the time resolution $\Delta \tau^{4}$ used in our observations) which is equal to $\Delta \tau / \tau\left(\nu_{3}, \nu_{4}\right)=5.5 / 10^{4}$, and second by the profile morphology at $16.699 \mathrm{MHz}$. The morphology analysis shows that the fiducial point can be determined with an accuracy of about the adopted resolution, $\pm 1.5 \Delta \tau$. Thus the error is $\pm 1.5 \Delta \tau / \tau\left(\nu_{a}, \nu_{4}\right)=8.36 / 10^{4}$, which when recalculated in terms of the $D M$ 
gives $\Delta D M_{\mathrm{real}}= \pm 0.0045 \mathrm{pc} \mathrm{cm}^{-3}$. This corresponds to Smirnova et al. (1986) rather well. Note that the attempt to use the data from Kuz'min et al. (1985) for finding the $D M$ according to the methods discussed leads to the conclusion that we are dealing with a complex geometry of the pulsar magnetosphere rather than with the effects of aberration and twist.

Though the discussion given above is quite correct, it would be desirable to conduct simultaneous multifrequency observations in order to exclude the effects of electron density fluctuations in interstellar medium and kinematic corrections connected with the Earth motions. Therefore together with V. M. Malofeev and Yu. P. Shitov, our colleagues from the Radio Astronomy Observatory of the Lebedev Physical Institute, we conducted simultaneous multifrequency observations of PSR 0809+74 on 19-25 Jan 1987 using the radiotelescopes BSA, DKR 1000 (Pushchino) and UTR-2 (Grakovo). The observations were conducted on the initiative of our colleagues to confirm the detection and the quantitative measurement of the STD effect earlier reported (Shitov 1985). The results obtained were presented as a report at the XIX All-Union Conference on Galactic and Extragalactic Radioastronomy in Tallinn, 1987. Shitov et al. (1988) partially reported on the observations using the BSA and DKR-1000 arrays. Here we present the best profiles obtained on $20 \mathrm{Jan} 1987$ at $\nu_{2}, \nu_{3}=24.846 \mathrm{MHz}$ and $\nu_{4}=16.436 \mathrm{MHz}$ and the maximum positions of the average profile at $\nu_{1}=102.746 \mathrm{MHz}$ obtained with the BSA (figure 2). The following time delays were obtained: $\tau_{\mathrm{A}}\left(\nu_{1}, \nu_{2}\right)=36.419545 \mathrm{~s}, \tau_{\mathrm{B}}\left(\nu_{1}, \nu_{2}\right)=36.4987 \mathrm{~s}, \tau_{\mathrm{A}}\left(\nu_{3}, \nu_{4}\right)=49.705027 \mathrm{~s}$, $\tau_{\mathrm{B}}\left(\nu_{3}, \nu_{4}\right)=49.7314 \mathrm{~s}$. The simultaneity accuracy of the conjoint observations was $4 \mathrm{~ms}$ and accounting for the profile morphology, the error was defined by the UTR-2 resolution of $\tau=26 \mathrm{~ms}$. Substitution of the data from these observations into eq.(1) gives for the fiducial points $A$ and $B$ in figure 2 the corresponding values:

$$
D M(\mathrm{~A})_{\text {real }}=5.753 \pm 0.003 \mathrm{pc} \mathrm{cm}^{-3} ; D M(\mathrm{~B})_{\text {real }}=5.751 \pm 0.003 \mathrm{pc} \mathrm{cm}^{-3}
$$

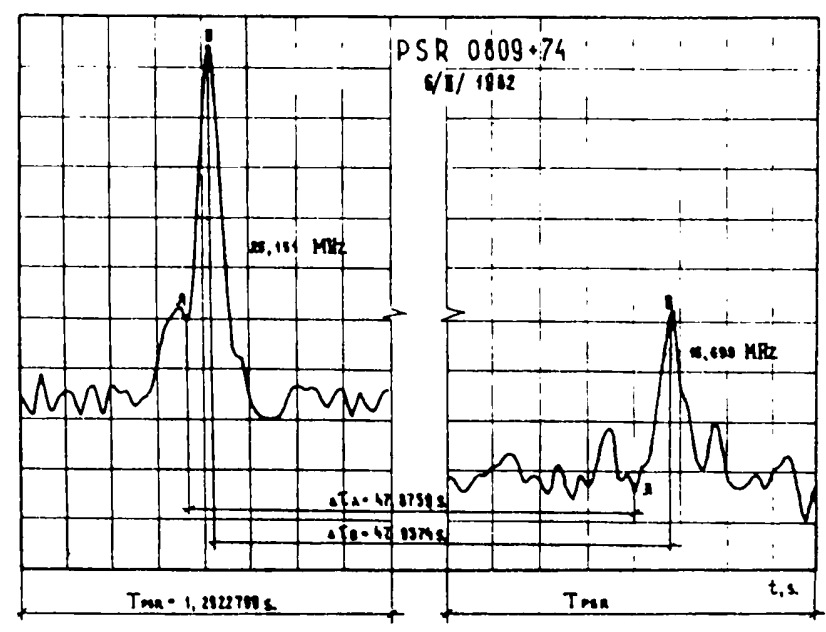

Figure 1 Observations of PSR $0809+74$ on 5 and 6 November 1982

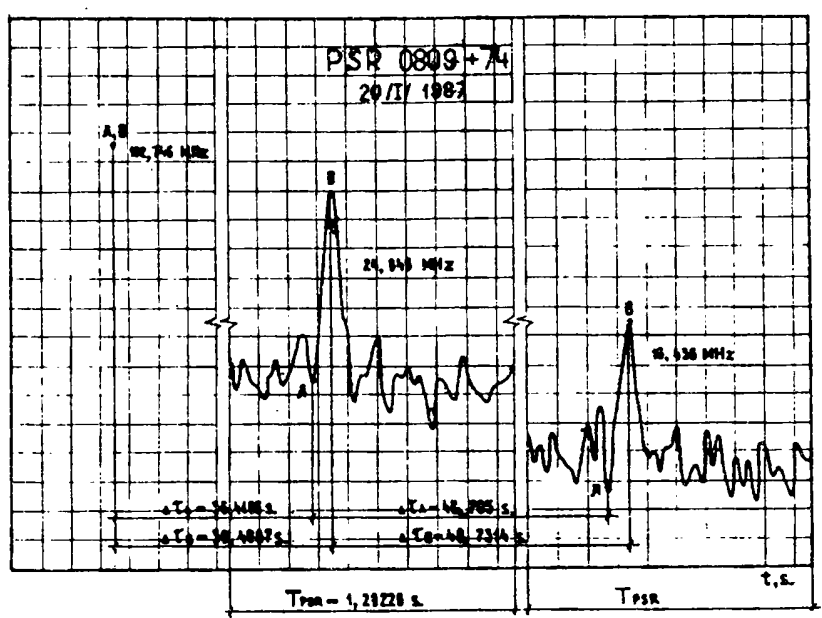

Figure 2 Profiles of PSR $0809+74$ obtained 20 January 1987

These considerations enable us to make the following conclusions:

1. The most accurate methods of determining the $D M$ for the low dispersion pulsar PSR $0809+74$ result in an error of $\Delta D M= \pm 0.003 \mathrm{pc} \mathrm{cm}^{-3}$;

2. In this connection, there is no sense in speaking about the detection of the twist effect of the magnetic field lines for PSR 0809+74 and higher dispersion pulsars (Shitov 1985, Shitov, Malofeev, and Izvekova 1988, Kuz'min 1985a) based on the observed time delays of the fiducial points less than $\Delta D M\left(1 / \nu_{i}^{2}-1 / \nu_{j}^{2}\right) / 2.41$;

3. Simultaneous multifrequency observations of the average profiles of pulsars may yield only an upper limit on the magnitude of the aberration effect prevailing for the small radii radiation as compared to the twist effect. 